\title{
Reflets
}

Revue ontaroise d'intervention sociale et communautaire

\section{Maintien à domicile francophone}

\section{Richard Martel et Carole Pinsonneault}

Volume 2, numéro 2, automne 1996

Vieillir à l'aube de l'an 2000

URI : https://id.erudit.org/iderudit/026138ar

DOI : https://doi.org/10.7202/026138ar

Aller au sommaire du numéro

Éditeur(s)

Reflets : Revue ontaroise d'intervention sociale et communautaire

ISSN

1203-4576 (imprimé)

1712-8498 (numérique)

Découvrir la revue

Citer cet article

Martel, R. \& Pinsonneault, C. (1996). Maintien à domicile francophone. Reflets,

2(2), 150-157. https://doi.org/10.7202/026138ar

Tous droits réservés (C) Reflets : Revue ontaroise d'intervention sociale et communautaire, 1996

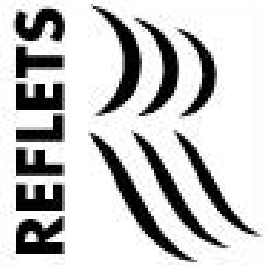

Ce document est protégé par la loi sur le droit d'auteur. L'utilisation des services d’Érudit (y compris la reproduction) est assujettie à sa politique d'utilisation que vous pouvez consulter en ligne.

https://apropos.erudit.org/fr/usagers/politique-dutilisation/ 


\title{
Maintien à domicile francophone
}

\author{
Richard Martel et Carole Pinsonneault \\ Centre médico-social communautaire (CMSC), Toronto
}

Dans une quinzaine d'années, les premiers bébés d'après la deuxième guerre mondiale (baby boomers) atteindront l'âge de 65 ans. On prévoit donc une augmentation substantielle des gens âgés par rapport au reste de la population. Le système de santé ontarien doit être remanié pour faire face aux demandes de soins que provoquera l'accroissement de cette clientèle. Il faut retarder le plus longtemps possible la prise en charge des personnes âgées par des institutions, afin de diminuer un fardeau fiscal qui deviendrait vite insoutenable pour les contribuables.

À l'instar des autres provinces canadiennes, et depuis une dizaine d'années, le gouvernement de l'Ontario a entrepris une réforme des soins de longue durée. Il est prévu que dans les régions désignées par la Loi 8 sur les services en français, la planification des soins de longue durée doit reconnaitre et appuyer l'engagement d'offrir à la population francophone des services dans la langue de son choix.

Il y a actuellement de 5000 à 6000 personnes francophones de plus de 65 ans sur le territoire du Toronto métropolitain, ce qui représente environ $10 \%$ des francophones présents. Selon les projections du ministère de la santé de l'Ontario, elles seront près de 9000 en l'an 2010.

\section{Le seul organisme francophone de santé}

Le Centre médico-social communautaire (CMSC) de Toronto est 
le seul organisme structuré qui offre des soins de santé en français sur le territoire du Toronto métropolitain. Il était donc normal qu'il s'intéresse de près à la Réforme des soins de longue durée du gouvernement ontarien. Fondé en 1989, le cMSc jalonne sa courte histoire par de nombreuses réalisations visant l'amélioration de l'état de santé et des conditions sociales de la population francophone de la Communauté urbaine de Toronto. La gamme des services offerts actuellement s'étend de la médecine familiale aux soins infirmiers en passant par la diététique, l'interprétation médicale, la santé mentale et l'intervention communautaire. Le CMSC est considéré par les autorités gouvernementales comme le représentant des besoins des francophones du grand Toronto en matière de santé et de services sociaux

\section{Le CMSC au cœur de la réforme}

C'est à partir de 1991 que le CMSC s'est joint, avec d'autres organismes du milieu, au mouvement de réforme des soins aux personnes âgées. C'est un domaine de haute importance puisque les francophones âgés de la région de Toronto n'ont pas accès dans leur langue à des soins primaires et des soins à domicile structurés.

Vu la pénurie de données disponibles sur les personnes âgées francophones du Toronto métropolitain, le CMSC a obtenu du financement auprès du ministère de la Santé de l'Ontario, afin d'étudier les conditions socio-économiques de ces francophones et les services de santé auxquels ils ont accès. Les résultats de l'étude, combinés aux données générales recueillies par le ministère de la Santé de l'Ontario, par Statistiques Canada et par le ministère fédéral de la Santé, ont servi de base à l'élaboration, par le CMSC, du projet de maintien à domicile pour les personnes âgées francophones. 


\section{Déterminants de la santé des personnes âgées}

Au cours de l'été 1995, le CMSC a réalisé l'Étude sur la santé des personnes aînées qui résident sur le territoire du Toronto métropolitain (Beaulieu et Lemire, 1995). Grâce à un sondage effectué auprès de 78 personnes de 65 ans et plus, le CMSC a pu recueillir des données sur le revenu, le mode d'habitation, les conditions sociales, les loisirs, l'utilisation actuelle des services de santé, etc.

Ces renseignements sont de grande importance pour déterminer l'état de santé d'une population. L'Enquête sur la santé des Ontariens de 1990, effectuée par le ministère de la Santé de l'Ontario, établit que le revenu, l'éducation, le soutien social (la famille) et l'emploi sont des facteurs déterminants de la santé (1992).

\section{Conditions sociales peu enviables}

Les conditions sociales des personnes âgées francophones du Toronto métropolitain sont peu enviables, avec en moyenne un revenu annuel plus bas que celui des anglophones (46\% des francophones vivraient sous le seuil de la pauvreté). Par rapport aux anglophones, ces personnes doivent dépenser une proportion plus élevée de leur revenu au logement $(14 \%$ de plus si elles sont propriétaires, $11 \%$ de plus si elles sont locataires). Selon les données de Statistique Canada (1991), 68 \% des aînés canadiens sont propriétaires de leur habitation. L'étude du CMSC indique qu'en moyenne, seules $40 \%$ des personnes âgées francophones sont propriétaires de leur habitation.

L'accès aux loisirs semble aussi plus limité. L'étude du CMSC révèle qu'en majorité les personnes âgées interrogées dépensent moins de 20 \$ par semaine aux loisirs. La majorité de la population âgée canadienne y consacre plus de 20 \$ par semaine. 
De plus, selon l'étude du CMSC, 73 \% des personnes âgées francophones consomment régulièrement des médicaments. Ce pourcentage est de $38 \%$ chez les personnes âgées canadiennes. Les données recueillies sur l'état de santé actuel des personnes âgées du Toronto métropolitain indiquent un grave problème d'hypertension (48\%). De plus, elles montrent un taux élevé de dépres$\operatorname{sion}^{1}(26 \%)$, de tabagisme (22\%), de consommation d'alcool, la prévalence du diabète $(17 \%)$ et une grande consommation de médicaments $(73 \%)$. Il s'agit véritablement d'une population à risques élevés. Ceci est confirmé par d'autres études canadiennes et ontariennes qui montrent que ces facteurs de risque sont plus élevés chez les francophones que chez les Canadiens en général.

\section{L'éducation garantit-elle la santé?}

Une bonne éducation ne garantit pas la santé, mais elle prédispose tout de même les personnes qui en ont bénéficié à adopter des comportements favorables à la santé. Le lien entre l'éducation et la santé se traduit par la capacité de mettre en pratique des décisions informées pour améliorer, maintenir et recouvrer la santé.

Un niveau d'éducation peu élevé est en soi un facteur de risque, surtout s'il est associé à la consommation excessive d'alcool, de tabac, à l'inactivité physique, à l'embonpoint, à un niveau élevé de stress et de dépression et, par le fait même, à l'utilisation accrue des services de santé. Selon Statistique Canada (1991), 70 \% des Canadiens ayant fait des études postsecondaires évaluent leur santé de bonne à excellente, par rapport à $46 \%$ des Canadiens qui n'ont pas terminé leurs études secondaires. En majorité, les personnes âgées francophones du Toronto métropolitain n'ont pas terminé d'études secondaires, et $39 \%$ n'ont pas terminé d'études primaires (Beaulieu et Lemire, 1995). 
Divers facteurs expliquent cette situation. Rappelons seulement qu'en Ontario, l'accès à l'éducation dans leur langue maternelle a toujours été difficile pour les francophones. La période la plus sombre fut de 1910 à 1927 où la mise en vigueur du Règlement 17 par le gouvernement de l'Ontario restreignait l'enseignement en français dans les écoles publiques et séparées. L'application du règlement a été vigoureusement contestée, et souvent contournée, par les parents canadiens-français. Il n'en demeure pas moins que l'accès à l'éducation était moins facile pour les FrancoOntariens que pour les Anglo-Ontariens.

Tout projet de soins aux personnes âgées francophones doit tenir compte du pourcentage élevé d'analphabétisme. Les gestionnaires de programmes de promotion de la santé et de services à domicile doivent privilégier une approche personnalisée pour rejoindre cette clientèle et l'aider à adopter des comportements favorables à la santé.

\section{Besoins identifiés}

Soixante-treize pour cent $(73 \%)$ des personnes âgées souhaitent que les services de santé en français soient décentralisés. Seul le CMSC offre, en français, des services de santé structurés dans la région de Toronto, et aucun hôpital ne garantit aux francophones des services dans leur langue.

Les francophones sont pourtant de grands utilisateurs des services de santé. Par exemple, $86 \%$ des répondants à l'enquête du CMSC avaient consulté un médecin au cours des six mois précédant le sondage. La moyenne canadienne pour cette catégorie de la population est de $90 \%$ sur un an.

Les conclusions sont évidentes. Actuellement, les personnes âgées francophones de Toronto n'ont pas accès dans leur langue à un réseau de services et de soins spécialisés. Elles ont pourtant besoin de ces services pour maintenir leur santé. Le ministère de 
la Santé de l'Ontario considère en effet l'accès aux services comme l'un des déterminants de la santé.

\section{Solution : des services à domicile}

Afin d'améliorer leur santé et leurs conditions de vie, le CMSC propose deux types d'initiatives aux personnes âgées: intervention à domicile et intervention communautaire et sociale. Toutes les deux s'inscrivent dans le projet Maintien à domicile francophone élaboré par le CMSC.

Le premier volet, les services de maintien à domicile, implique la mise sur pied de services de santé de base à domicile: services de médecins et de personnel infirmier pour toutes les personnes âgées francophones. De plus, un réseau de professionnels itinérants de la santé (diététiste, personnel infirmier, ergothérapeutes, psychothérapeutes, etc.) sera aussi créé pour offrir des soins de longue durée aux personnes âgées qui ne peuvent se déplacer. Des services de soutien personnel (aide pour prendre ses médicaments, s'habiller, se laver, etc.) et d'aide familiale (entretien ménager, cuisine, etc.) compléteront la gamme de services offerts. Cette approche multidisciplinaire permettra de renforcer les comportements santé prescrits par les professionnels et d'encourager de bonnes habitudes de vie.

Pour améliorer l'environnement social, le projet de Maintien à domicile francophone comprend des services de soutien communautaire : organisation de loisirs, de repas communautaires et de danses, etc. Ces services seront offerts en partenariat avec des organismes déjà actifs dans la communauté, comme la Corporation des Centres d'Accueil Héritage. Briser l'isolement des personnes âgées s'avère très important pour l'équilibre social de la personne. De plus, la pauvreté est souvent reliée à l'isolement, d'où la nécessité de mettre sur pied des campagnes de prévention et d'éducation en matière de santé. 


\section{Services intégrés}

Un gestionnaire de cas coordonnera l'ensemble des services nécessaires au bien-être de la clientèle. La centralisation de la gestion des services simplifiera l'accès téléphonique, permettra une meilleure évaluation des besoins et un suivi global.

Dans ce contexte, la création d'un dossier informatisé (DI) placera en interaction toutes les données concernant un seul client. Une vingtaine de services différents et de fournisseurs de soins sont présents dans le processus de maintien à domicile. Les modèles suisses et québécois du DI ont fait leurs preuves, et il est possible de les adapter à peu de frais au projet du CMSC.

\section{Bonne volonté}

Le CMSC propose de mettre à exécution le projet de Maintien à domicile francophone en l'intégrant à sa structure actuelle, solution pratique et peu coûteuse. Toutes les informations ont été transmises au ministère de la Santé de l'Ontario, qui étudie le projet.

Les services de maintien à domicile s'avèrent une nécessité, compte tenu de l'absence actuelle de services en français, des besoins clairement identifiés et des facteurs de risques pour la santé des personnes âgées francophones. Le tout doit se faire dans le respect de l'intégrité des personnes âgées, et en s'assurant la participation des organismes communautaires du milieu.

Des exemplaires de l'étude et du projet seront bientôt disponibles. Pour se les procurer, les personnes intéressées doivent en faire la demande par écrit au Centre médico-social communautaire, 22, rue College, Toronto (Ontario), M5G 1K3. 


\section{Note}

1. Le taux élevé de dépression est basé sur le taux canadien qui est de $26 \%$, et $46 \%$ des personnes interrogées par le CMSC ont indiqué que le counselling familial et individuel est leur priorité.

\section{Bibliographie}

BEAULIEU, M. et D. LEMIRE (1995). Études sur la santé des personnes ainées qui résident sur le territoire du Toronto métropolitain, Toronto, Centre médico-social communautaire de Toronto.

CENTRE MÉDICO-SOCIAL COMMUNAUTAIRE DE TORONTO (CMSC) (1996).

Le Maintien à domicile francophone - Projet de services de santé, de soins de longue durée, de services sociaux et communautaires pour les personnes âgées francophones du Toronto métropolitain, Toronto, Centre médico-social communautaire de Toronto.

MINISTÈRE DE LA SANTÉ DE L'ONTARIO (1992). L'enquête sur la santé des Ontariens, Faits saillants, Toronto, Gouvernement de l'Ontario.

STATISTIQUES CANADA (1991). L'état de santé des Canadiens, Enquête sociale générale, Ottawa, Gouvernement du Canada. 